\title{
Rapid Transition of an Allied Health Clinic to Telehealth During the COVID-19 Pandemic: Satisfaction and Experience of Health Professionals, Student Practitioners, and Patients
}

Michelle A. Krahe

Griffith University - Australia, m.krahe@griffith.edu.au

Maya Conway

Griffith University - Australia, m.conway@griffith.edu.au

Simone Howells

Griffith University, Australia, s.howells@griffith.edu.au

Kara Roffey

Griffith University - Australia, k.roffey@griffith.edu.au

Sheena Reilly

Griffith University - Australia, pvchealth@griffith.edu.au

Follow this and additional works at: https://nsuworks.nova.edu/ijahsp

Part of the Medicine and Health Sciences Commons

\section{Recommended Citation}

Krahe MA, Conway M, Howells S, Roffey K, Reilly S. Rapid Transition of an Allied Health Clinic to Telehealth During the COVID-19 Pandemic: Satisfaction and Experience of Health Professionals, Student Practitioners, and Patients. The Internet Journal of Allied Health Sciences and Practice. 2021 Jan 01;19(3), Article 9.

This Manuscript is brought to you for free and open access by the College of Health Care Sciences at NSUWorks. It has been accepted for inclusion in Internet Journal of Allied Health Sciences and Practice by an authorized editor of NSUWorks. For more information, please contact nsuworks@nova.edu. 


\title{
Rapid Transition of an Allied Health Clinic to Telehealth During the COVID-19 Pandemic: Satisfaction and Experience of Health Professionals, Student Practitioners, and Patients
}

\begin{abstract}
Purpose: With COVID-19 social distancing measures requiring a shift in how healthcare is delivered, telehealth service provision allows patients to receive care remotely while adhering to relevant safety regulations. This study investigated the perceptions and experiences of allied health practitioners and their patients at a multidisciplinary allied health clinic that rapidly transitioned to telehealth service delivery. Method: Allied health practitioners (both qualified and student) and patients were recruited during the transition to telehealth across a large healthcare facility located on the Gold Coast, Australia. Participants were surveyed after each telehealth session rating their satisfaction and experience across four areas (technical, administrative and operational, privacy, communication). Descriptive statistics were used to express categorical variables and a chi-square test of independence was applied to determine the presence of any associations. Results: Surveys $(n=197)$ were collected from allied health practitioners $(n$ $=31$ ) and their patients $(n=70)$. Overall, high levels of satisfaction and experience with telehealth among patients and a consensus in satisfaction and experience among exercise physiology, physiotherapy and psychology practitioners were reported. Speech pathology student practitioners rated their satisfaction and telehealth experience significantly $(p<0.001)$ lower than other disciplines. A significant relationship $\left[\chi^{2}(2, n=127)=7.49, p=.02\right]$ between student practitioners and the impact of telehealth on achieving session goals and outcomes was also identified. Conclusions: This study highlights complexities related to the acceptability and adoption of telehealth, technical aspects, and user functionality which contribute to the growing body of evidence supporting digital health technologies in the delivery and access to allied health services. In a multidisciplinary context, this study advocates for the consideration of disciplinespecific issues when designing and implementing digital health services.
\end{abstract}

\section{Author Bio(s)}

Dr Michelle Krahe, PhD, BAppSci (BiomedSci) (Hons) is a Senior Health Research Fellow at Griffith University with extensive experience in clinical and primary health research. She has a record of leadership and expertise in research strategy, development and management in the research, academia, government and health sectors.

Maya Conway MPH, MSpPath, CPSP is a researcher in the Health Group and School of Allied Health Sciences at Griffith University. Maya is a certified speech-language pathologist with a background in public health. Her areas of expertise include child health, speech-language pathology and service delivery for vulnerable populations.

Dr Simone Howells PhD, BSpPath(Hons), GradCertClinEd, CPSP is a Lecturer and Clinical Education Coordinator in the Speech Pathology Program, School of Allied Health Sciences, Griffith University. She is a certified practicing speech pathologist with expertise in adult dysphagia and the education and training of speech pathology students.

Kara Roffey BExSc/BPhty, MSportsPhysio, is the Director of the Allied Health and Psychology Clinic at Griffith University's Gold Coast campus in Queensland, Australia. She is also a senior physiotherapist with expertise in sports physiotherapy.

Professor Sheena Reilly AM, FASSA FAHMS FSPA FRCSLT is Pro Vice Chancellor (Health) at Griffith University and Australia's foremost academic in childhood communication disorders. She is among the most highly cited researchers in her field, devoting 30-years of her career to understanding speech, 
language and literacy development in children.

\section{Acknowledgements}

We would like to acknowledge the Griffith University Health Clinic and allied health professionals and patients who participated in this study. 


\title{
IIIAHSP \\ The Internet Joumnal of Allied Health Sciences and Practice \\ Dedicated to allied health professional practice and education
}

Vol. 19 No. 3 ISSN 1540-580X

\section{Rapid Transition of an Allied Health Clinic to Telehealth During the COVID-19 Pandemic: Satisfaction and Experience of Health Professionals, Student Practitioners, and Patients}

\author{
Michelle A. Krahe \\ Maya Conway \\ Simone Howellls \\ Kara Roffey \\ Sheena Reilly \\ Griffith University
}

Australia

\begin{abstract}
Purpose: With COVID-19 social distancing measures requiring a shift in how healthcare is delivered, telehealth service provision allows patients to receive care remotely while adhering to relevant safety regulations. This study investigated the perceptions and experiences of allied health practitioners and their patients at a multidisciplinary allied health clinic that rapidly transitioned to telehealth service delivery. Method: Allied health practitioners (both qualified and student) and patients were recruited during the transition to telehealth across a large healthcare facility located on the Gold Coast, Australia. Participants were surveyed after each telehealth session rating their satisfaction and experience across four areas (technical, administrative and operational, privacy, communication). Descriptive statistics were used to express categorical variables and a chi-square test of independence was applied to determine the presence of any associations. Results: Surveys ( $n=197$ ) were collected from allied health practitioners $(n=31)$ and their patients $(n=70)$. Overall, high levels of satisfaction and experience with telehealth among patients and a consensus in satisfaction and experience among exercise physiology, physiotherapy and psychology practitioners were reported. Speech pathology student practitioners rated their satisfaction and telehealth experience significantly $(p<0.001)$ lower than other disciplines. A significant relationship $\left[X^{2}(2, n=127)=7.49, p\right.$ $=.02]$ between student practitioners and the impact of telehealth on achieving session goals and outcomes was also identified. Conclusions: This study highlights complexities related to the acceptability and adoption of telehealth, technical aspects, and user functionality which contribute to the growing body of evidence supporting digital health technologies in the delivery and access to allied health services. In a multidisciplinary context, this study advocates for the consideration of discipline-specific issues when designing and implementing digital health services.
\end{abstract}

Key words: telehealth, telemedicine, allied health, multidisciplinary, COVID-19, health service evaluation 


\section{INTRODUCTION}

In March 2020, the WHO declared the novel coronavirus (COVID-19) a global pandemic. ${ }^{1}$ Because of the highly contagious nature of this virus, strict hygiene measures and social distancing requirements were quickly enacted. As a result, healthcare providers needed to implement a way of providing remote access to services for patients requiring ongoing care, which gave rise to the significant expansion of telehealth service delivery. ${ }^{2-6}$ Telehealth is a delivery model that involves the use of various information and communication technologies (ICTs) and is generally facilitated between several health professionals or an interaction between a health practitioner and a patient. ${ }^{7}$ The former allows practitioners to provide/receive peer mentoring or seek specialist support, the latter facilitates patient care (e.g., for chronic conditions, management of medications, discharge or follow up, counselling and other health needs).

Telehealth removes geographical barriers, allowing patients to attend appointments from the comfort of their home or local facilities within their community. ${ }^{8-10}$ While it boasts many positive benefits, it also carries complex challenges. Research indicates that limited ICT experience combined with low patient health literacy is a major factor contributing to a patient's inability or unwillingness to participate in telehealth. ${ }^{11}$ Other reported barriers include the inability to access appropriate ICTs, lack of adequate internet connection, confidentiality and/or privacy concerns, linguistic or cultural variances, and the associated costs. ${ }^{12-14}$ Despite these challenges, progression towards telehealth service provision is advocated in an increasingly digitised world. ${ }^{15-17}$

Australia's allied health professionals represent more than one-quarter of the health workforce and deliver an estimated 200 million health services annually. ${ }^{18} \mathrm{It}$ is a diverse sector with significant variation across and within professions, providing care for people of all ages with chronic illnesses or mental ill-health, and those experiencing disability. Only in the last decade has telehealth emerged as a means of providing allied health services..$^{19-22}$ The literature cites numerous reasons that have influenced or delayed the uptake in Australia, including practitioner's lack of e-health skills and equipment, concerns about risks to patient privacy, perceived reduction in productivity, and concerns regarding detrimental impacts to patient-practitioner relationships..$^{21}$ These factors, along with evidence suggesting patient's preference for in-person consultations, add to the complexity in the uptake of telehealth for allied health services. ${ }^{21}$ As such, to mitigate barriers and enhance enablers, it is important to understand telehealth service provision within the allied health sector. This is particularly important within the Australian context, as evidence of allied health service provision via telehealth is limited. ${ }^{21,23}$

The purpose of this study was to evaluate the rapid transition to telehealth delivery of a multidisciplinary allied health service from practitioner and patient perspectives. This is the first study to investigate the use of telehealth during the COVID-19 pandemic in Australia that specifically involves several allied health disciplines and includes student practitioners. It is hoped that the outcomes of this research will contribute to a growing body of evidence surrounding the use of digital health technologies to increase and improve access to care.

\section{METHODS}

\section{Study Design and Setting}

This study employed a cross-sectional, observational design and sampling technique. It was conducted in partnership with the Griffith University Health Clinics (referred herein as the "Clinic"), a large healthcare facility located on the Gold Coast, Australia. The Clinic offers a range of multidisciplinary health services (i.e., dentistry, dietetics, exercise physiology, physiotherapy, psychology, speech pathology, and social work) to the local community. In 2019, before the COVID-19 pandemic, approximately 3,000 members of the public attended a service at the Clinic, in a one-off or ongoing treatment capacity. Services were delivered by qualified practitioners as well as supervised student practitioners in a face-to-face treatment capacity. Services were charged to patients at rates set by the Clinic and determined by the type and duration of service provided.

During the COVID-19 pandemic in 2020, the Clinic rapidly transitioned to telehealth delivery of multidisciplinary allied health care. This study investigates patient and practitioner perceptions and experiences of telehealth at the Clinic. Feedback was obtained through an online survey from allied health practitioners (both qualified and student practitioners), and members of the public (patients) participating in telehealth delivered services. During the study period, telehealth services were delivered synchronously, and charges were subsidised by the Australian Government (free or reduced charge for the patient). The patient and practitioner communicated using videoconferencing (i.e., two-way audio and visual capabilities) via Microsoft Teams for assessment and/or intervention activities (similar to those provided during traditional in-person sessions). This study was approved by the Human Research Ethics Committee of Griffith University (2020/357).

\section{Participants and Recruitment}

Participants were recruited over an eight-week period (1 June to 29 July 2020), coinciding with the rapid transition to telehealth across the Clinic. Qualified and student practitioners from the allied health disciplines of dietetics, exercise physiology, 
physiotherapy, psychology, speech pathology, and social work were invited to participate. Student practitioners received basic technical training in the use of the telehealth platform, watched videos of telehealth sessions related to their discipline and received some telehealth adapted therapy resources from their clinical educators (qualified practitioners). Students were supervised by qualified practitioners while providing telehealth services. Qualified practitioners also received technical training and were supported to upskill through their respective professional bodies All individuals who participated in a telehealth session during the study period were eligible; those aged $<18$ years were eligible only if an adult or guardian was present during the session and agreed to complete the survey with them, or on their behalf. Patient participation was voluntary, and it is anticipated that the subsidised cost of telehealth sessions was favourable to recruitment.

\section{Data Collection}

After each telehealth session, participants were invited to complete an online survey. The survey was hosted online via an open-source application (LimeSurvey - GmbH, v1.9X, Hamburg Germany), which could be accessed using both mobile and desktop devices. Allied health practitioners were provided with a link via email from the Clinic Manager, and patients received a survey link via the chat function or via email following their telehealth session. All participants were provided with an information sheet detailing the study and completion of the survey was established as consent.

\section{Survey Development}

The survey was designed through an iterative process with the study investigators and was based on a review of existing literature.22,24-26 Key themes for evaluation included technological literacy, the intrusiveness of the technology, user interface design, simplicity and usability of the technology, integration of telehealth with existing workflow, ease of implementation, and readiness to change. ${ }^{24}$ There were two versions of the survey; one for practitioners (Appendix A) and one for patients (Appendix B), with slight variations in questions grouped into four domains: 1) participant characteristics, 2) experience, 3) service satisfaction, and 4) service experience. The survey was pre-tested with a group of allied health practitioners who were invited to give their opinion regarding the survey length and question clarity. This feedback was incorporated into the final surveys. The length of the survey was intentionally brief, as evidence suggests this improves online survey response rate, quality, and attentiveness. ${ }^{27}$ Skip logic was utilised to encourage survey completion. For the response rate calculation, surveys with at least $80 \%$ completion were included in analysis.

\section{Data Analysis}

The main outcome measures were derived from survey questions regarding service satisfaction and service experience with telehealth. These questions were rated on a five-point Likert scale and the weighted mean \pm standard deviation (SD) is presented. Descriptive statistics were used to express categorical variables as counts and percentages. Where data are not normally distributed, median and interquartile range (IQR) were obtained. The response options to satisfaction (question 3.1) and experience (questions 4.1 to 4.4) questions were grouped into three categories for analysis; satisfaction: (1) unsatisfied (very and somewhat), (2) neutral (neither satisfied nor unsatisfied), and (3) satisfied (somewhat and very); experience: (1) disagree (somewhat and strongly), (2) neutral (neither agree nor disagree), and (3) agree (somewhat and strongly). These data are presented as diverging stacked bar charts. ${ }^{28}$

All analyses were conducted using SPSS statistics (version 25.0, IBM). A chi-square $\left(\chi^{2}\right)$ test of independence was performed to examine the presence of associations. Compliance with assumptions was checked using cross-tabulations and significant interactions were reported. In cases where these assumptions were violated, exact tests (Bonferroni) were used to confirm the $p$-value. A significance threshold of $p<0.01$ was adopted in all analyses to compensate for the unrecorded confounders that are more likely to exist in observational research. ${ }^{29}$ Due to the small number of exercise physiology practitioners and patients, they were not able to be included in the cross-discipline analysis.

\section{RESULTS}

\section{Allied Health Practitioners}

Of the allied health disciplines invited to participate in the study, responses from exercise physiology, physiotherapy, psychology, and speech pathology were obtained. In total, 31 practitioners (16.1\%; $n=5$ qualified and $83.9 \% ; n=26$ student practitioners) participated in the study. This represents a response rate of $61 \%$ (31 out of 51). The average practitioner age was $29.2 \pm 8.0$ years (range 22 to 55) (Table 1). Most practitioners had previously worked in the Clinic and had experience using telehealth. There were only six practitioners (all students) who had never worked in the Clinic and had never delivered services by telehealth previously. The time taken to complete the survey was 1.4 minutes (IQR $1.0-2.3$ minutes). 
Table 1. Characteristics and experience of allied health practitioners, as distributed by qualified, student and total. Values represent count and proportion (\%) of the column total unless otherwise indicated.

\begin{tabular}{|lcc|c|}
\hline \multirow{2}{*}{ Variable } & \multicolumn{3}{c|}{ Allied Health Practitioners } \\
\cline { 2 - 4 } & Qualified & $\begin{array}{c}\text { Student } \\
\mathrm{n}(\%)\end{array}$ & $\begin{array}{c}\text { Total } \\
\mathrm{n}(\%)\end{array}$ \\
\hline Participants & $5(16.1)^{\star}$ & $26(83.9)^{\star}$ & $31(60.8)$ \\
Age years, mean \pm SD & $30.0 \pm 9.0$ & $29.2 \pm 8.0$ & $29.2 \pm 8.0$ \\
Allied health discipline & & & \\
$\quad$ Exercise Physiology & $1(20.0)$ & $2(7.7)$ & $3(9.7)$ \\
$\quad$ Physiotherapy & $1(20.0)$ & $9(34.6)$ & $10(32.3)$ \\
$\quad$ Psychology & $3(60.0)$ & $9(34.6)$ & $12(38.7)$ \\
$\quad$ Speech Pathology & 0 & $6(23.1)$ & $6(19.4)$ \\
First time providing a service in the Clinic? & & & \\
$\quad$ Yes & $1(20.0)$ & $9(34.6)$ & $10(32.2)$ \\
No & $4(80.0)$ & $17(65.4)$ & $21(67.8)$ \\
First time delivering a telehealth service? & & & \\
Yes & 0 & $9(34.6)$ & $9(29.0)$ \\
No & $5(100)$ & $17(65.4)$ & $22(71.0)$ \\
First time Clinic and telehealth service? & & & $6(19.4)$ \\
$\quad$ Yes & 0 & $20(77.0)$ & $25(80.6)$ \\
No & $5(100)$ & &
\end{tabular}

*Values are expressed as a proportion (\%) of the total.

In total, 143 surveys were received from allied health practitioners; of which 16 containing incomplete data were excluded. Of the remaining 127 surveys, $49.6 \%(n=63)$ were from physiotherapy, 29.1\% $(n=37)$ psychology, 18.1\% $(n=23)$ speech pathology, and $3.1 \%(n=4)$ exercise physiology. The proportion of surveys completed by student practitioners $(n=107)$ was significantly greater than those completed by qualified practitioners $(n=20)$, which was reflective of the Clinic service delivery during this time $(p<0.001)$.

Overall, $68.5 \%(n=87)$ of practitioners were satisfied with the delivery of services by telehealth $(3.7 \pm 0.7)$ (Appendix C). When analysed by discipline, there was consensus among exercise physiology, physiotherapy and psychology practitioners; a significantly greater proportion of speech pathology practitioners were unsatisfied with telehealth $(47.8 \% ; p<0.001)$ compared to physiotherapy (1.6\%) and psychology (5.4\%) (Figure 1). The proportion of speech pathology practitioners who were satisfied with telehealth $(39.1 \%)$ was also significantly less than physiotherapy $(71.4 \%)$ and psychology $(81.0 \%)$ practitioners.

The adequacy and acceptability of the technical and administrative aspects of delivering services by telehealth were viewed positively $(63 \%, 3.5 \pm 0.6 ; 76.4 \%, 4.0 \pm 0.8)$ overall (Figure $2 \mathrm{~A}$ and B). There were some significant differences between disciplines, with a greater proportion of speech pathology practitioners who reported disagreement with the technical and administrative aspects of telehealth. Overall, practitioners mostly agreed that the use of telehealth did not impact their ability to facilitate positive patient-practitioner interactions $(64 \% ; 3.6 \pm 0.6)$ and that telehealth did not inhibit their ability to achieve session goals and/or expected clinical outcomes (64\%; $3.6 \pm 0.6$ ) (Figure 2C and 2D). Responses from speech pathology practitioners were significantly different to those received from physiotherapy and psychology practitioners, specifically regarding impacts on patient-practitioner interactions and the ability to achieve session goals. A chi-square test of independence showed an association between student practitioners and the perceived impact of telehealth on session goals, $\chi^{2}(2, N=127)=7.4, p=.02$. Qualified practitioners were more likely than student practitioners to agree that telehealth did not impact their ability to achieve session goals and/or clinical outcomes. 


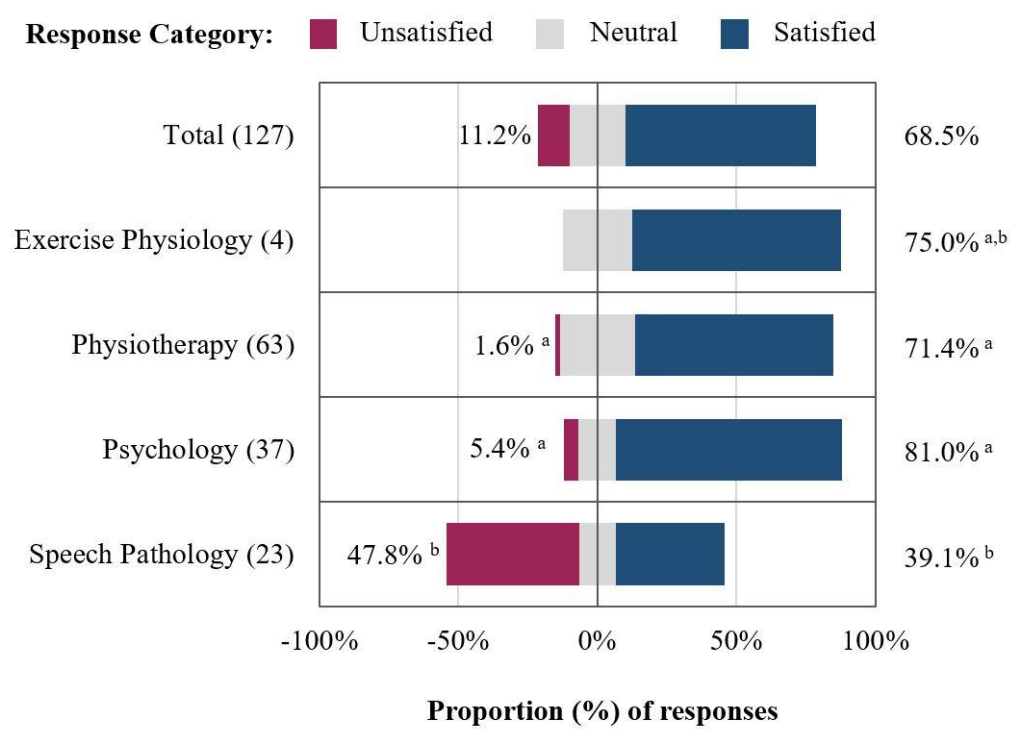

Figure 1. Overall satisfaction with telehealth service delivery by allied health practitioners. Numbers in brackets show absolute numbers of respondents and percentages are given for unsatisfied and satisfied response categories. Different superscript letters denote significant differences at $p<0.01$.

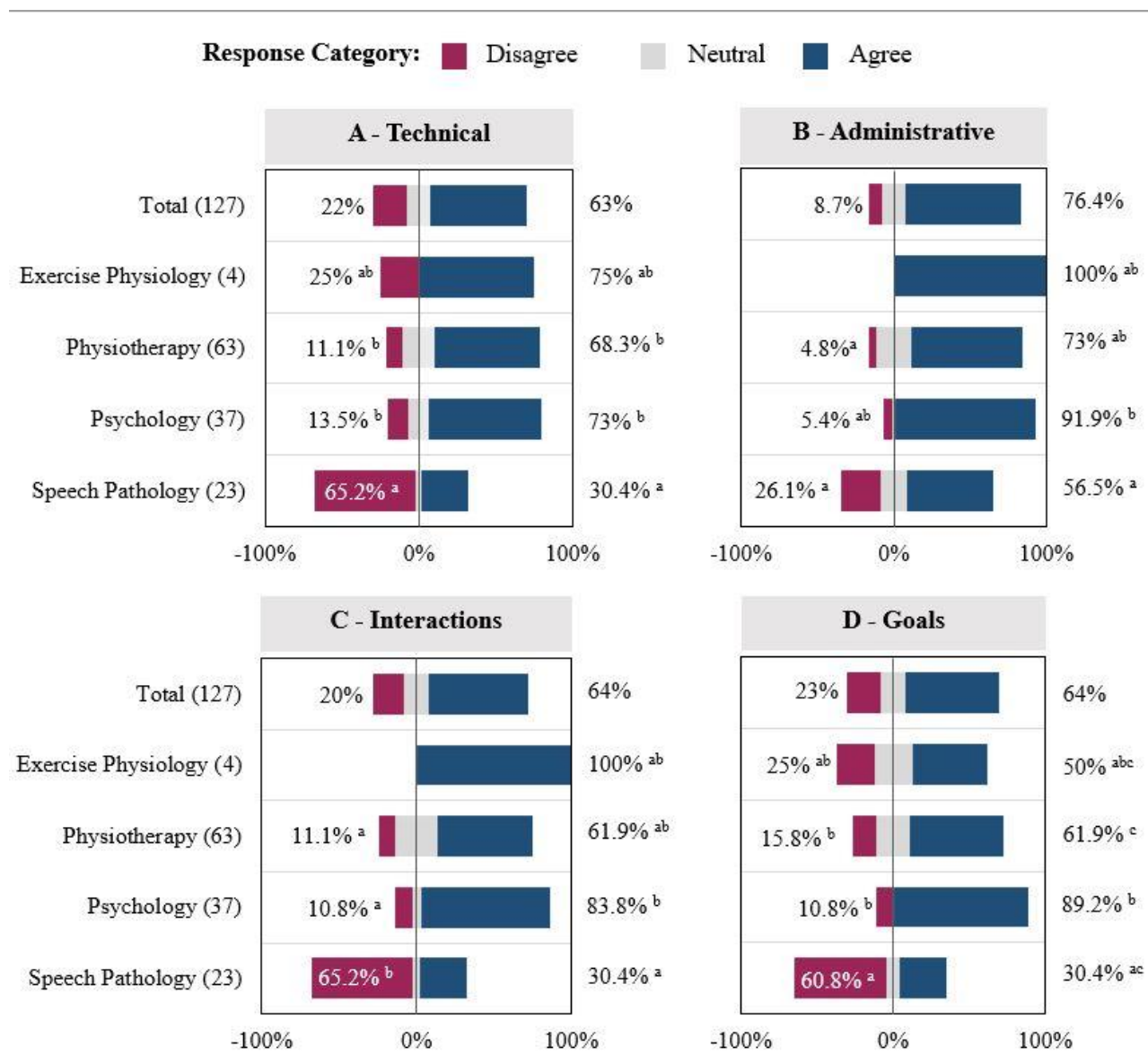

Figure 2A-D. Allied health practitioner evaluation of whether the (A) technical and $(B)$ administrative aspects of telehealth were adequate and acceptable, and whether telehealth did not impact the ability to (C) facilitate positive patient-practitioner interactions, and (D) achieve session goals/expected clinical outcomes. Numbers in brackets show absolute numbers of respondents and percentages are given for disagree and agree response categories. Different superscript letters within the same response category denote significant differences at $p<0.01$. 


\section{Allied Health Patients}

During the recruitment period, 240 individuals participated in a telehealth service at the Clinic. In total, 129 surveys were received; of which 59 were excluded ( 38 were incomplete and 21 were returning patients), for a final response rate of $29 \%$ (70 out of 240). Of the 70 patients who completed surveys, the majority were female $(64.3 \% ; n=45)$ and younger than 18 years of age (32.9\%; $n=23)$ (Table 2). There were significantly more patients under the age of 18 years than any other age group $(p<0.001)$. A chi-square test of independence confirmed that there was a significant association between patient's age $\left(\chi^{2}(18, N=70)=72.9, p<0.001\right)$, whether their service was provided by a student practitioner $\left(\chi^{2}(3, N=70)=29.9, p<\right.$ $0.001)$ and the allied health discipline. There were significantly more patients who attended speech pathology sessions that were younger than 18 years, compared to all other disciplines and significantly more student practitioners who provided physiotherapy and speech pathology services (Table 2). Most participants had previously attended the Clinic $(52.9 \%, n=37)$, but $57.1 \%(n=40)$ had never participated in telehealth. The time taken to complete the survey was 2.6 minutes (IQR $1.8-$ 5.0 minutes).

Table 2. Characteristics and experience of allied health patients, as distributed by allied health discipline and total. Values represent count and proportion (\%) of the column total unless otherwise indicated.

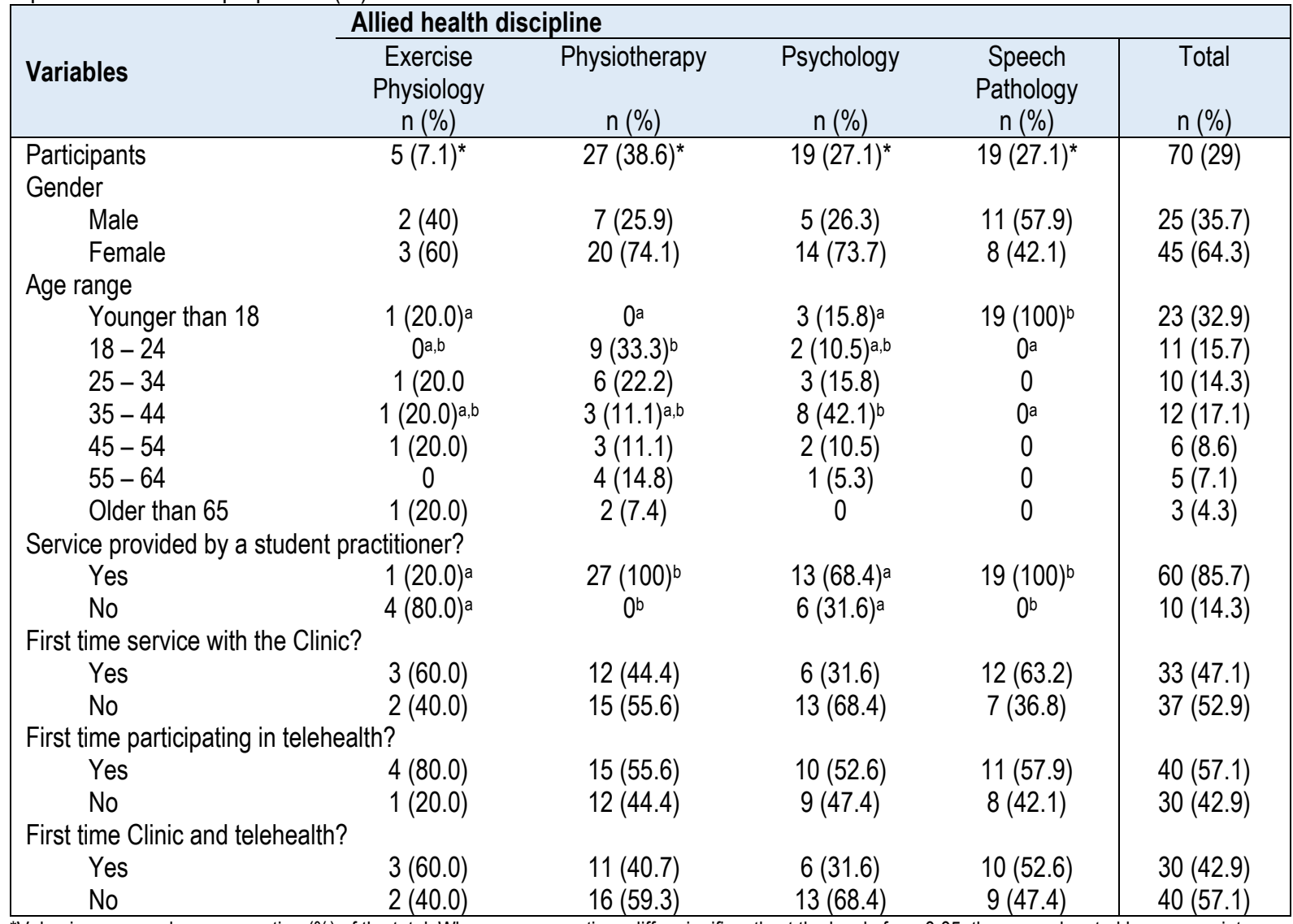

${ }^{*}$ Value is expressed as a proportion (\%) of the total. Where row proportions differ significantly at the level of $p<0.05$, these are denoted by superscript letters.

The majority of participants $(92.8 \%)$ were satisfied with their service provided by telehealth $(4.5 \pm 0.3)$ (Appendix D); one $(1.4 \%)$ speech pathology patient was unsatisfied and four $(5.8 \%)$ were neutral $(n=2$ speech pathology and $n=2$ psychology) (Figure 3). When analysed by discipline, there was an overwhelming consensus of satisfaction (exercise physiology $4.8 \pm 0.3$; physiotherapy $4.7 \pm 0.3$; psychology $4.5 \pm 0.2$; speech pathology $4.3 \pm 0.2$ ). There were no significant differences in satisfaction between patients receiving a service from a qualified practitioner or a student practitioner $(4.7 \pm 0.3 \mathrm{vs} .4 .5 \pm 0.3)$ and satisfaction was not influenced by patient gender $(p=0.39)$, age $(p=0.80)$, first time service with the Clinic $(p=0.39)$, or first time participating in telehealth $(p=0.75)$. 


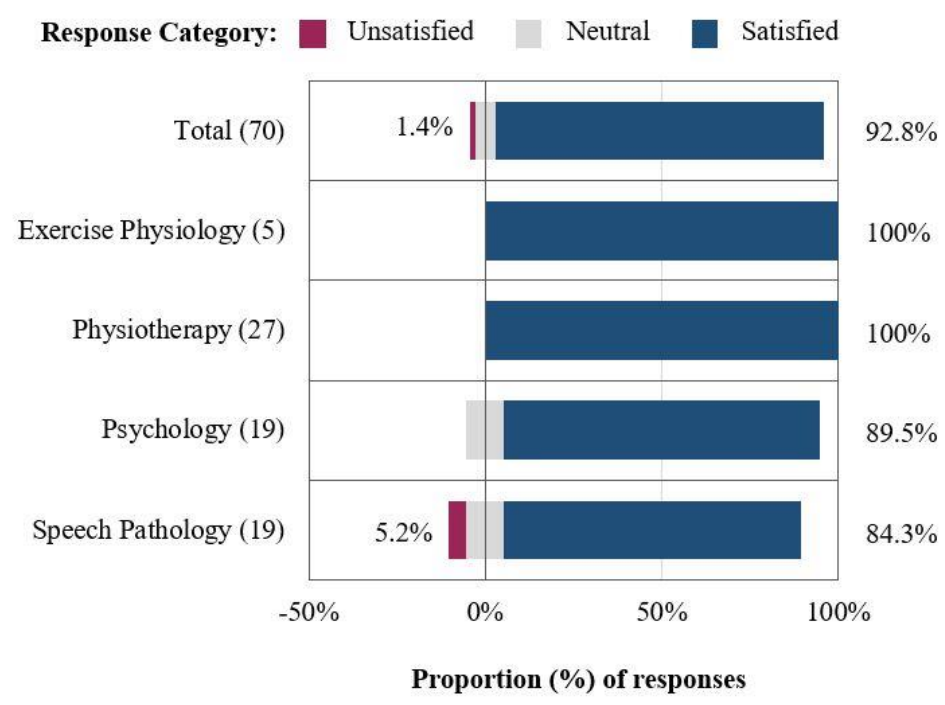

Figure 3. Overall satisfaction with telehealth service delivery by allied health patients. Numbers in brackets show absolute numbers of respondents and percentages are given for unsatisfied and satisfied response categories.

Patients agreed that the technical $(80 \%, 4.0 \pm 0.5)$ and administrative $(91.4 \%, 4.5 \pm 0.5)$ aspects of their telehealth service were adequate and acceptable (Figure $4 \mathrm{~A}$ and $4 \mathrm{~B}$ ), their privacy $(91.4 \%, 4.6 \pm 0.5)$ was adequately addressed and their expectations of the session were met $(94.3 \%, 4.7 \pm 0.5)$ (Figure $4 \mathrm{C}$ and 4D). There were no significant differences between patient responses across allied health disciplines.

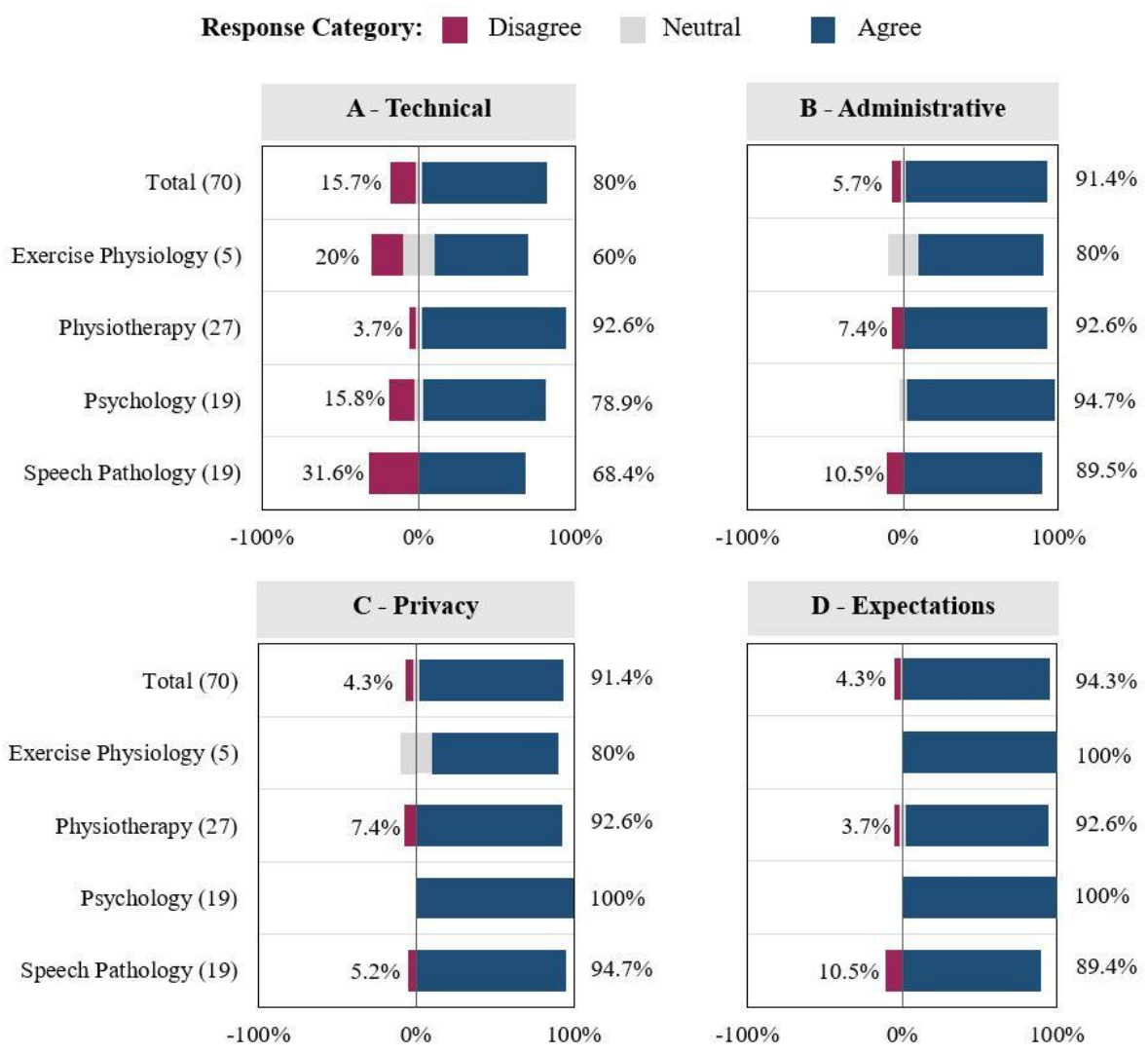

Figure 4A-D. Allied health patient evaluation of whether the $(A)$ technical, $(B)$ administrative and $(C)$ privacy and confidentiality aspects of telehealth were adequate and acceptable, and if (D) expectations of the session were met. Numbers in brackets show absolute numbers of respondents and percentages are given for disagree and agree response categories. 


\section{DISCUSSION}

With COVID-19 social distancing measures requiring a shift in how healthcare is delivered, the implementation and uptake of telehealth services have allowed patients to receive care remotely while adhering to these regulations. ${ }^{30,31}$ While the use of telehealth has been explored in healthcare more generally, there is limited evidence associated with multidisciplinary allied health services. ${ }^{32}$ There is also a gap in the literature featuring evaluations that capture both patient and practitioner perspectives and/or those contextualised within a rapid transition to telehealth.

This novel study investigated the rapid transition to telehealth delivery of a multidisciplinary allied health service, evaluating the satisfaction and experience of both qualified and student practitioners, and their patients. Our results show that within the allied health disciplines of exercise physiology, physiotherapy, psychology and speech pathology, telehealth is an acceptable way to deliver/receive care. The analysis allows for increased understanding of elements related to system usability, perceived user experience, patient-practitioner interactions, and clinical goal attainment. Moreover, this study highlights the influence of allied health practitioner and patient characteristics, such as discipline nuances, experience and expertise, and digital literacy. These aspects need to be considered and addressed as this model of service delivery is scaled.

Overall satisfaction with telehealth delivery was high among allied health practitioners and their patients, despite many never having used it previously. These findings are consistent with the broader telehealth literature; albeit the contributing factors differ. In a systematic review and narrative analysis of patient satisfaction of telehealth, patient health outcomes and improvements in physical and behavioural conditions were primarily associated with higher satisfaction. ${ }^{33}$ Others have reported patient satisfaction to be associated with ease of use, low cost or cost savings and preferred modality, however these studies centre around measures of effectiveness and efficiency of novel telehealth modalities. ${ }^{34-36}$ In our study, satisfaction generally did not differ among the allied health disciplines; except for speech pathology student practitioners and this difference is of questionable clinical significance given that their patients indicated high satisfaction with the services they received. This is more likely to be associated with specific elements of the telehealth application and patient characteristics, which are discussed in more detail below.

All patient discipline groups reported extremely high rates of agreement with the adequacy of the technical (i.e., sound and picture quality, connectivity, set-up, and ease of use) and administrative elements (i.e., scheduling, rebooking, reception, and instructions/assistance for use of the technology) of their telehealth session. This was also the case for patient acceptability towards their privacy and confidentiality being met, as well as their expectations of the session. Despite there being no significant differences among patient responses, a larger proportion rated the technological aspects of telehealth least favourable, albeit only $16 \%$. Issues included problems with sound and picture quality, connectivity delays, and difficulties retrieving shared content - one patient recalls "... throughout our sessions the video hangs, there's usually a 2-5 second delay when the host switches to the shared screen graphics, and often at key points when [the practitioner is] trying to show the way to say something, the video hangs and it has to be repeated...". This finding aligns with previous research citing poor video and audio quality, slow internet speeds and difficulty navigating or using systems resulting in barriers to telehealth and negative user experiences. ${ }^{13}$ Also noteworthy, were the proportion of patients from exercise physiology and physiotherapy who did not agree or were neutral as to whether their privacy and confidentiality were adequately addressed. An explanation for this may be the physical nature of these disciplines and the necessity to conduct visual examinations of affected body parts which may require the patient to remove items of clothing, wear specific fitted clothing or position themself in a way that is conducive to a physical exam.

Overall, most allied health practitioners agreed that the technical and administrative aspects of delivering services by telehealth were adequate and acceptable and that telehealth did not impact their ability to have positive patient interactions or achieve the intended clinical/session goals. Results from our evaluation confirm that practitioners made positive transitions to telehealth delivery of allied health services. Except for technical aspects, psychology practitioners and patients reported the highest level of satisfaction and agreement across all areas of evaluation; these findings are reflected widely in the literature. ${ }^{37}$ Psychology as a discipline may lend itself well to the use of either video and/or audio mediums due to the largely verbal nature of therapies where physical examination and physical touch is less required. As such, patients attending telehealth psychology sessions may feel more comfortable with the process knowing that there are no significant changes to the way they receive therapy other than the lack of the physical therapy environment which interestingly, did not appear to impact patients' perceptions of acceptability with privacy and confidentiality of their sessions.

For the discipline evaluation of telehealth experience, (i.e., technology, patient interactions and achieving session goals), there were significantly more speech pathology practitioners who responded negatively ('disagree') compared to physiotherapy and/or psychology disciplines. Practitioners who reported that the telehealth technology was not acceptable identified issues related to connectivity, software and patient capacity to use the technology - one practitioner recalls "...patient had trouble using Teams and kept losing my video, so it was hard to demonstrate exercises"... These findings are supported by research 
which advocates for familiarity with technology to facilitate faster adaption and greater acceptance. ${ }^{13}$ With the Clinic's rapid transition to telehealth due to a global pandemic, it is feasible that practitioners and patients may not have had enough exposure/training for the applications used and due to the diversity of the allied health disciplines, the technological requirements may have differed greatly. For example, speech pathology services require the ability to share screen control with patients to facilitate learning activities, while physiotherapy requires more advanced visual capabilities, often with a portable camera. It should also be considered that these two disciplines were primarily delivered by student practitioners.

Contrary to other disciplines, student practitioners providing speech pathology services reported that telehealth impacted their ability to facilitate positive patient interactions and achieve session goals. This finding must also be considered in the context of the speech pathology patient caseload at the Clinic, which was exclusively paediatric ( $<18$ years) in contrast to the patient base seen by all other disciplines. Collaborating, building rapport and keeping young children engaged when using only telehealth mediums has unique challenges. ${ }^{32}$ While there is research to suggest there is no difference between telehealth and in-person sessions on paediatric patient interaction, results from our study suggest otherwise but should be considered within the context of the practitioner's experience. ${ }^{38}$ Despite being supervised by qualified practitioners, student's may lack general experience and expertise in their field, and the addition of a new service delivery model, particularly during a time of high stress, would be challenging. Further, as previously highlighted, the technological challenges associated with telehealth including the inability of the telehealth program (Microsoft Teams) to facilitate two-way screen control was reported to add to the challenges engaging paediatric patients in learning activities. Telehealth's inability to facilitate physical touch, which can be critical for some elements of therapy, and indeed speech pathology, may also reduce the capacity of practitioners to attain the results sought ${ }^{32}$ Under these circumstances, it is not surprising that more experienced practitioners may be more readily able to adapt to the telehealth model. Conversely, while patients generally reported that their expectations of the session were met, practitioner perspectives were more negative. This suggests that evaluating outcome measures together with service experience might provide a more objective indicator of expected clinical outcome.

Unlike other studies which examine telehealth, the current study limits selection bias since all enrolled participants were offered telehealth services. However, when the Clinic began to transition to telehealth, patients were required to opt-in to receive this service, which may have inadvertently introduced some selection bias to our sample. A strength of the study is the collection of both allied health practitioner and patient feedback, providing a more comprehensive evaluation to better inform our understanding of telehealth service delivery. The smaller sample size and participant homogeneity should be considered limitations, and likely contribute to the high satisfaction reports. Additionally, small numbers of participants from exercise physiology meant that statistical analysis was not able to be completed for this group. Further, the high proportion of student practitioners participating in the study warrants consideration when interpreting the results and limits the generalisability of patient-practitioner experience data; albeit, the inclusion of student perspectives is also a unique aspect of the study. Future research in the area should continue to examine satisfaction and acceptability and adoption over a longer timeframe, on a greater scale, with larger and randomly selected participants to understand patterns and possible influencing factors. While this study focused on specific allied health disciplines, the findings may also have broader application for the design and implementation of telehealth services among other allied health disciplines or clinical settings.

Providing continuity of care; especially in the context of the COVID-19 pandemic, telehealth helped to maintain allied health services and follow social distancing guidelines for practitioners and patients at the Clinic. This early evaluation demonstrates high levels of satisfaction and positive experiences from both patients and practitioners. It also highlights complexities related to the acceptability and adoption of telehealth, technical aspects, and user functionality/capability. The potential to expand telehealth delivery of allied health services is obvious, as are the benefits for practitioners, patients, and student skill development; however, consideration of discipline-specific issues is important in the design and implementation of services post-pandemic. As a result of this research, telehealth has now become a core service offered by the Clinic for patients preferring this method of service delivery. Further, allied health courses at Griffith University now include an embedded telehealth module to support student skill development. It is hoped that this study may be useful for other multidisciplinary allied health organisations considering a transition to telehealth or those seeking further insights into existing services.

\section{CONFLICTS OF INTEREST}

The Author(s) declare(s) that there is no conflict of interest.

\section{ACKNOWLEDGMENTS}

We would like to acknowledge the Griffith University Health Clinic and the allied health professionals and patients who participated in this study.

\section{FUNDING}

This research received no specific grant from any funding agency in the public, commercial, or not-for-profit sectors. 


\section{REFERENCES}

1. World Health Organization (WHO). Responding to community spread of COVID-19. 2020.

2. World Health Organization (WHO). Maintaining essential health services: operational guidance for the COVID-19 context. 2020.

3. Hollander JE, Carr BG. Virtually perfect? Telemedicine for COVID-19. N Engl J Med. 2020;382(18):1679-1681.

4. Reeves JJ, Hollandsworth HM, Torriani FJ, et al. Rapid response to COVID-19: health informatics support for outbreak management in an academic health system. J Am Med Inform Assoc. 2020;27(6):853-859.

5. Schulz T, Long K, Kanhutu K, Bayrak I, Johnson D, Fazio T. Telehealth during the coronavirus disease 2019 pandemic: rapid expansion of telehealth outpatient use during a pandemic is possible if the programme is previously established. J Telemed Telecare. 2020.

6. Smith AC, Thomas E, Snoswell CL, et al. Telehealth for global emergencies: Implications for coronavirus disease 2019 (COVID-19). Journal of telemedicine and telecare. 2020;26(5):1357633X2091656-1357313.

7. Tuckson RV, Edmunds M, Hodgkins ML. Telehealth. N Engl J Med. 2017;377(16):1585-1592.

8. Moffatt JJ, Eley DS. The reported benefits of telehealth for rural Australians. Aust Health Rev. 2010;34(3):276-281.

9. Bradford NK, Caffery LJ, Smith AC. Telehealth services in rural and remote Australia: a systematic review of models of care and factors influencing success and sustainability. Rural and Remote Health. 2016;16(4):3808.

10. Uscher-Pines $L$, Thompson J, Taylor $P$, et al. Where virtual was already reality: the experiences of a nationwide telehealth service during the COVID-19 pandemic. J Med Internet Res. 2020;22(12):22727.

11. Kim H, Xie B. Health literacy in the eHealth era: a systematic review of the literature. Patient Educ Couns. 2017;100(6):1073-1082.

12. Scott Kruse C, Karem P, Shifflett K, Vegi L, Ravi K, Brooks M. Evaluating barriers to adopting telemedicine worldwide: a systematic review. J Telemed Telecare. 2018;24(1):4-12.

13. Almathami HKY, Win KT, Vlahu-Gjorgievska E. Barriers and facilitators that influence telemedicine-based, realtime, online consultation at patients' homes: systematic literature review. J Med Internet Res. 2020;22(2):e16407.

14. Dorsey ER, Topol EJ. State of telehealth. N Engl J Med. 2016;375(2):154-161.

15. Kvedar J, Coye MJ, Everett W. Connected health: a review of technologies and strategies to improve patient care with telemedicine and telehealth. Health Affairs. 2014;33(2):194-199.

16. World Health Organization (WHO). Telemedicine - opportunities and developments in Member States: report on the second global survey on eHealth. Geneva: World Health Organization;2010. 2220-5462.

17. Mehrotra A, Ray K, Brockmeyer DM, Barnett ML, Bender JA. Rapidly converting to "virtual practices": outpatient care in the era of COVID-19. N Engl J Med Catalyst. 2020.

18. Allied Health Professions Australia. What is allied health? https://ahpa.com.au/what-is-allied-health/. Published 2020. Accessed 11 November, 2020.

19. Speyer R, Denman D, Wilkes-Gillan S, et al. Effects of telehealth by allied health professionals and nurses in rural and remote areas: a systematic review and meta-analysis. J Rehabil Med. 2018;50(3):225-235.

20. Guilfoyle C, Wootton R, Hassall S, et al. User satisfaction with allied health services delivered to residential facilities via videoconferencing. Journal of Telemedicine and Telecare. 2003;9(1):52-54.

21. lacono T, Stagg K, Pearce N, Hulme Chambers A. A scoping review of Australian allied health research in ehealth. BMC Health Serv Res. 2016;16(1):543.

22. Queensland Health. Evaluation resource guide: allied health telehealth capacity building project. 2016.

23. Allied Health Professions Australia. Submission 208 Select Committee on COVID-19: inquiry into the Australian Government's response to the COVID-19 pandemic 2020.

24. Agboola S, Hale TM, Masters C, Kvedar J, Jethwani K. "Real-world" practical evaluation strategies: a review of telehealth evaluation. JMIR Res Protoc. 2014;3(4):e75.

25. Doyle C, Lennox L, Bell D. A systematic review of evidence on the links between patient experience and clinical safety and effectiveness. BMJ Open. 2013;3(1):e001570.

26. Brear M. Evaluating telemedicine: lessons and challenges. Health Inf Manag. 2006;35(2):23-31.

27. Galesic M, Bosnjak M. Effects of questionnaire length on participation and indicators of response quality in a web survey. Public Opinion Quarterly. 2009;73(2):349-360.

28. Heiberger RM, Robbins NB. Design of diverging stacked bar charts for Likert scales and other applications. J Stat Softw. 2014;57(5):1-32.

29. von Elm E, Altman DG, Egger M, et al. The strengthening the reporting of observational studies in epidemiology (STROBE) statement: guidelines for reporting observational studies. Int J Surg. 2014;12(12):1495-1499.

30. Mann DM, Chen J, Chunara R, Testa PA, Nov O. COVID-19 transforms health care through telemedicine: evidence from the field. J Am Med Inform Assoc. 2020;27(7):1132-1135.

31. Chunara R, Zhao Y, Chen J, et al. Telemedicine and healthcare disparities: a cohort study in a large healthcare system in New York City during COVID-19. J Am Med Inform Assoc. 2020;28(1):33-41. 
32. Campbell J, Theodoros D, Russell T, Gillespie N, Hartley N. Client, provider and community referrer perceptions of telehealth for the delivery of rural paediatric allied health services. Aust J Rural Health. 2019;27(5):419-426.

33. Scott Kruse C, Krowski N, Rodriguez B, Tran L, Vela J, Brooks M. Telehealth and patient satisfaction: a systematic review and narrative analysis. BMJ Open. 2017;7(8):e016242.

34. Georgsson M, Staggers N. Quantifying usability: an evaluation of a diabetes mHealth system on effectiveness, efficiency, and satisfaction metrics with associated user characteristics. J Am Med Inform Assoc. 2016;23(1):5-11.

35. Minatodani DE, Chao PJ, Berman SJ. Home telehealth: facilitators, barriers, and impact of nurse support among high-risk dialysis patients. Telemed J E Health. 2013;19(8):573-578.

36. Jacobs J, Ekkelboom R, Jacobs J, van der Molen T, Sanderman R. Patient satisfaction with a teleradiology service in general practice. BMC Fam Pract. 2016;17(1):17.

37. Connolly SL, Miller CJ, Lindsay JA, Bauer MS. A systematic review of providers' attitudes toward telemental health via videoconferencing. Clinical Psychology-Science and Practice. 2020;27(2):1-19.

38. Freckmann A, Hines M, Lincoln M. Clinicians' perspectives of therapeutic alliance in face-to-face and telepractice speech-language pathology sessions. Int J Speech Lang Pathol. 2017;19(3):287-296. 


\section{APPENDICES}

Appendix A. Telehealth evaluation survey completed by allied health practitioners.

\begin{tabular}{|clr|}
\hline$\#$ & Domain/Question & $\begin{array}{r}\text { Response } \\
\text { options }\end{array}$ \\
\hline 1 & Participant characteristics & Birth year \\
\hline 1.1 & Age & \\
\hline $\mathbf{2}$ & Experience & Yes / No \\
\hline 2.1 & Was this your first time providing a service in the Griffith University Health Clinics? & Yes / No \\
2.2 & Was this your first time delivering a telehealth service? & 6options \\
\hline 2.3 & Which service did you provide for this session? & Yes / No \\
\hline 2.4 & Are you a student practitioner? & Lpen \\
\hline 3 & Service satisfaction & \\
\hline 3.1 & Overall, how satisfied were you with telehealth as a mode of delivery for this service? \\
\hline 3.2 & Is there anything else you would like to share about your telehealth experience? & Likert 1-5b \\
\hline $\mathbf{4}$ & Service experience & Likert 1-5b \\
\hline Using the scale provided, rate your agreement with each of the following statements: & Likert 1-5b \\
4.1 & The technical aspects were adequate and acceptable & \\
4.2 & The administrative and operational aspects were adequate and acceptable \\
4.3 & The use of telehealth did not impact on my ability to facilitate positive interactions with the & Likert 1-5b \\
& patient & \\
4.4 & The use of telehealth did not impact on my ability to achieve session goals and/or the expected & clinical outcomes of this session.
\end{tabular}

a Likert scale ( $1=$ very unsatisfied, $2=$ somewhat unsatisfied, $3=$ neutral, $4=$ somewhat satisfied, $5=$ very satisfied). ${ }^{b}$ Likert scale ( 1 = strongly disagree, $2=$ somewhat disagree, $3=$ neutral, $4=$ somewhat agree, $5=$ strongly agree) . 
Appendix B. Telehealth evaluation survey completed by allied health patients.

\begin{tabular}{|c|c|c|}
\hline \# & Domain/Question & $\begin{array}{r}\text { Response } \\
\text { options }\end{array}$ \\
\hline 1 & Participant characteristics & \\
\hline 1.1 & Age range & 7 ranges \\
\hline 1.2 & Gender & 3 options \\
\hline 2 & Experience & \\
\hline 2.1 & Was this your first time using the Griffith University Health Clinic? & Yes / No \\
\hline 2.2 & Was this your first time participating in a telehealth delivered service? & Yes / No \\
\hline 2.3 & Which service did you receive today? & 6 options \\
\hline 2.4 & Was your service provided by a student practitioner? & Yes / No \\
\hline 3 & Service satisfaction & \\
\hline 3.1 & Overall, how satisfied were you with the service provided by telehealth? & Likert 1-5a \\
\hline 3.2 & Is there anything else you would like to share about your telehealth experience? & Open \\
\hline 4 & Service experience & \\
\hline \multicolumn{3}{|c|}{ Using the scale provided, rate your agreement with each of the following statements: } \\
\hline 4.1 & The technical aspects were adequate and acceptable & Likert 1-5b \\
\hline 4.2 & The administrative and operational aspects were adequate and acceptable & Likert 1-5b \\
\hline 4.3 & My privacy and confidentiality were adequately addressed & Likert 1-5b \\
\hline 4.4 & My expectations for the session were met & Likert 1-5b \\
\hline
\end{tabular}

a Likert scale ( $1=$ very unsatisfied, $2=$ somewhat unsatisfied, $3=$ neutral, $4=$ somewhat satisfied, $5=$ very satisfied). ${ }^{b}$ Likert scale ( 1 = strongly disagree, 2 = somewhat disagree, 3 = neutral, 4 = somewhat agree, 5 = strongly agree).

Appendix C. Likert scale responses and weighted means to main outcome measures for allied health practitioners.

\begin{tabular}{|rccccc|c|}
\hline \multirow{2}{*}{ Measure } & \multicolumn{5}{c}{ Response categories } & Mean \\
\cline { 2 - 5 } & $\mathbf{1}$ & $\mathbf{2}$ & $\mathbf{3}$ & $\mathbf{4}$ & $\mathbf{5}$ & $\mathbf{\pm}$ SD \\
\hline Satisfaction $^{\mathrm{a}}$ & 9 & 5 & 26 & 60 & 27 & $3.7 \pm 0.7$ \\
Experience $^{\mathrm{b}}$ & & & & & & \\
Technical & 9 & 19 & 19 & 58 & 22 & $3.5 \pm 0.6$ \\
Administrative & 2 & 9 & 19 & 45 & 52 & $4.0 \pm 0.8$ \\
Interactions & 10 & 16 & 20 & 47 & 34 & $3.6 \pm 0.6$ \\
Goals & 6 & 23 & 17 & 43 & 38 & $3.6 \pm 0.6$ \\
\hline
\end{tabular}

a Likert scale ( $1=$ very unsatisfied, $2=$ somewhat unsatisfied, $3=$ neutral, $4=$ somewhat satisfied, $5=$ very satisfied). ${ }^{\circ}$ Likert scale $(1=$ strongly disagree, 2 = somewhat disagree, $3=$ neutral, $4=$ somewhat agree, $5=$ strongly agree $)$. 
Appendix D. Likert scale responses and weighted means to main outcome measures for allied health patients.

\begin{tabular}{|c|c|c|c|c|c|c|}
\hline \multirow[b]{2}{*}{ Measure } & \multicolumn{5}{|c|}{ Response categories } & \multirow{2}{*}{$\begin{array}{l}\text { Mean } \\
\pm S D\end{array}$} \\
\hline & 1 & 2 & 3 & 4 & 5 & \\
\hline Satisfactiona & 0 & 1 & 4 & 21 & 44 & $4.5 \pm 0.3$ \\
\hline \multicolumn{7}{|l|}{ Experience $^{b}$} \\
\hline Technical & 0 & 11 & 3 & 27 & 29 & $4.0 \pm 0.5$ \\
\hline Administrative & 2 & 2 & 2 & 15 & 49 & $4.5 \pm 0.5$ \\
\hline Interactions & 2 & 1 & 3 & 9 & 55 & $4.6 \pm 0.5$ \\
\hline Goals & 1 & 2 & 1 & 8 & 58 & $4.7 \pm 0.5$ \\
\hline
\end{tabular}

a Likert scale (1 = very unsatisfied, 2 = somewhat unsatisfied, 3 = neutral, $4=$ somewhat satisfied, 5 = very satisfied). ${ }^{\mathrm{b}}$ Likert scale ( 1 = strongly disagree, 2 = somewhat disagree, 3 = neutral, $4=$ somewhat agree, 5 = strongly agree). 